\title{
Strong luminescence from dislocation-free GaN nanopillars
}

\author{
Y. Inoue ${ }^{\text {a) }}$ T. Hoshino, S. Takeda, K. Ishino, A. Ishida, and H. Fujiyasu \\ Department of Electrical and Electronic Engineering, Shizuoka University, 3-5-1 Johoku, \\ Hamamatsu 432-8561, Japan \\ H. Kominami, H. Mimura, and Y. Nakanishi \\ Research Institute of Electronics, Shizuoka University, 3-5-1 Johoku, Hamamatsu 432-8561, Japan \\ S. Sakakibara \\ Yamaha Corporation, 203 Matsunokijima, Toyooka-mura, Iwata-gun, Shizuoka 438-0192, Japan
}

(Received 14 May 2004; accepted 14 July 2004)

\begin{abstract}
$\mathrm{GaN}$ nanostructures were prepared on $\mathrm{Si}(111)$ by a hot-wall epitaxy technique employing the modified two-step growth method. Isolated hexagonal pillar-like $\mathrm{GaN}$ nanostructures (GaN nanopillars) with the typical diameter, height, and density of $200-300 \mathrm{~nm}, 0.5-1 \mu \mathrm{m}$, and 3-4 $\times 10^{8} \mathrm{~cm}^{-2}$, respectively, are self-organized without intentional pre-processing to the Si substrate. The photoluminescence and cathodoluminescence (CL) measurements show the strong near-band-edge emissions without the yellow band at room temperature. Stronger CL is obtained from the GaN nanopillars in comparison to single-crystalline GaN. The obtained strong CL is related to high crystal quality of the dislocation-free GaN nanopillars. (C) 2004 American Institute of Physics. [DOI: 10.1063/1.1792793]
\end{abstract}

In the low-voltage electron-impact excitation devices, a GaN-based phosphor for blue or UV light emitting sources attracts much interest. ${ }^{1}$ Since light emission is obtained by cathodoluminescence (CL) in such devices, it is not needed to fabricate a single-crystalline phosphor, and also not needed to control impurity doping. Moreover, emission intensity can be simply increased by increasing the excitation power of electron beam. However, since the penetration depth of electron beam of several kilovolts is as small as several tens of nanometer, light emission efficiency is affected by the crystal quality near the sample surface. Recently, strong photoluminescence (PL) from polycrystalline $\mathrm{GaN}$ grown on several kinds of substrates has been reported. ${ }^{2}$ The columnar GaN has also been reported, where the $\mathrm{GaN}$ is completely relaxed and emits intense and narrow PL. ${ }^{3,4}$ This strong emission could be related to the high crystallinity of each crystal segment. Such high emission from polycrystalline GaN should be a promising feature for developing GaN-based phosphors.

In this letter, we demonstrate the growth of faceted hexagonal pillar-like $\mathrm{GaN}$ nanostructures on $\mathrm{Si}$ for the development of UV light sources by a hot-wall epitaxy (HWE) technique. The structural and optical properties of the samples were characterized by scanning electron microscopy (SEM), transmission electron microscopy (TEM), PL and CL measurements. Isolated hexagonal pillar-like $\mathrm{GaN}$ nanostructures (GaN nanopillars) are self-organized without artificial preprocessing to the substrates. The GaN nanopillars are highly luminescent and show stronger CL emission in comparison to the single-crystalline continuous $\mathrm{GaN}$ films grown on a sapphire substrate. The cross-sectional TEM observation reveals that such effective luminescence is related to the growth of dislocation-free crystals.

The growth of GaN was performed in a HWE system, which was a simple method for the growth of compound semiconductors, and their superlattices. ${ }^{5,6}$ The reagent source

a)Electronic mail: yoku@hwe.eng.shizuoka.ac.jp materials for $\mathrm{Ga}$ and $\mathrm{N}$ were metallic gallium and $\mathrm{NH}_{3}$, respectively. GaN was grown by the modified two-step growth method. ${ }^{6,7} \mathrm{Si}(111)$ substrates were treated with buffered HF solution. No intentional pre-processing to the Si substrate was performed. First, the $\mathrm{Si}(111)$ substrate was initiated by nitridation at $930^{\circ} \mathrm{C}$. Then the substrate temperature $\left(T_{\text {sub }}\right)$ was lowered to $540^{\circ} \mathrm{C}$ and metallic $\mathrm{Ga}$ was deposited without $\mathrm{NH}_{3}$ gas flow. Following the Ga deposition, the Ga supply was shutted and the substrate was heated to $1020^{\circ} \mathrm{C}$ under the flowing of $\mathrm{NH}_{3}$. By this procedure, $\mathrm{GaN}$ seeds for the pillar-like growth were formed. The high temperature GaN growth was successively carried out at a $T_{\text {sub }}$ of $1020^{\circ} \mathrm{C}$ with the Ga supply and $\mathrm{NH}_{3}$ flow of $100 \mathrm{sccm}$.

The morphologies and crystal structures of the samples were studied using SEM. The optical properties were studied by $\mathrm{PL}$ excited by a $325 \mathrm{~nm} \mathrm{He}-\mathrm{Cd}$ laser with an excitation power of $1 \mathrm{~mW}$ and $\mathrm{CL}$ operated at $2 \mathrm{keV}$ with an electron beam current of $60 \mu \mathrm{A} / \mathrm{cm}^{-2}$. Both spot size of the laser and the electron beam were about $0.5 \mathrm{~mm}$ in diameter. In order to investigate the origin of strong luminescence, further structural properties were studied by cross-sectional TEM observation.

The plan and cross-sectional view SEM images of the GaN nanostructures are shown in Fig. 1. Clearly, faceted hexagonal pillar-like crystals are grown on the $\mathrm{Si}$ substrate with a large free surface area. Typical diameter and height are $200-300 \mathrm{~nm}$ and $0.5-1 \mu \mathrm{m}$, respectively, for $2 \mathrm{~h}$ growth. The diameter of pillars tends to widen from the bottom to the top. The density of the nanopillars is about 3-4 $\times 10^{8} \mathrm{~cm}^{-2}$. While the in-plane growth direction mostly agrees in each crystal, a small fraction of the nanopillars is inclined. In the cross-sectional SEM image [Fig. 1(b)], where the sample was cleaved along a $\mathrm{Si}(110)$ cleavage plane, flat side facets of GaN nanopillars are seen in the front. Since the ordered hexagonal pillar-like growth obviously originates from a wurtzite structure, the crystallographic relationship 


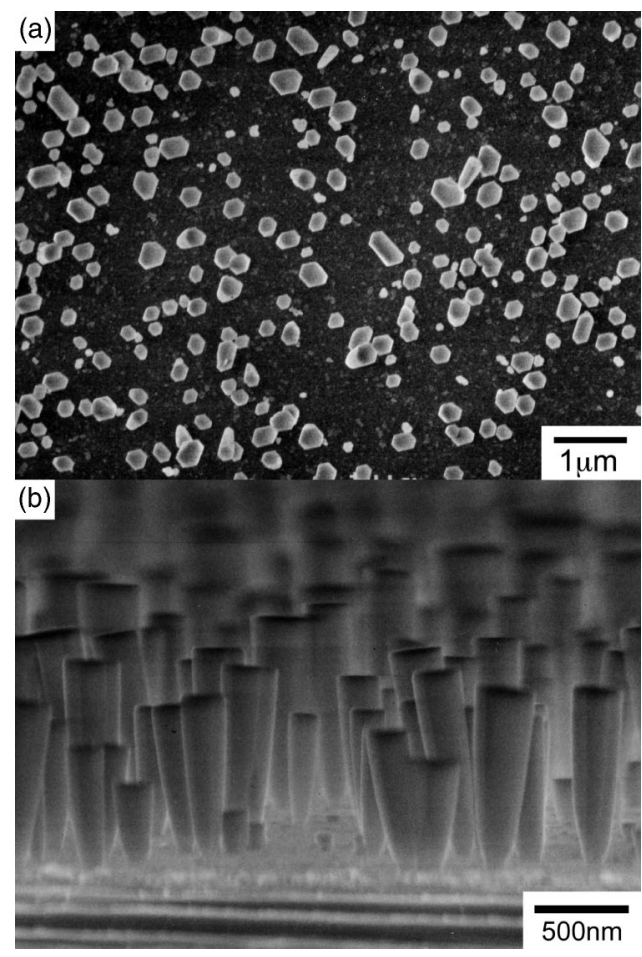

FIG. 1. (a) Plan and (b) cross-sectional view SEM images of the GaN nanopillars. For the cross-sectional observation, the sample was cleaved along a $\mathrm{Si}(110)$ plane.

between the $\mathrm{GaN}$ pillars and $\mathrm{Si}$ is $\mathrm{GaN}[0001] \| \mathrm{Si}[111]$, and $\operatorname{GaN}[1 \overline{1} 00] \| \operatorname{Si}[11 \overline{2}]$.

The columnar structures of GaN have been already reported and the columnar growth is typically obtained in highly mismatched systems. ${ }^{3,8-10}$ In the growth of isolated columnar structures, both low adsorbance of $\mathrm{Ga}$ on a substrate and formation of initial GaN seeds followed by the columnar growth would play an important role. Our case seems to satisfy such conditions. For the sample in the initial stage of the high temperature growth, GaN seeds, which sparsely spread with a similar density to GaN nanopillars, were observed by atomic force microscopy observation (not shown here). In the high temperature growth process, when evaporated $\mathrm{Ga}$ atoms reach to the $\mathrm{GaN}$ seed, they react and crystallize. On the other hand, when $\mathrm{Ga}$ atoms directly reach to a free Si surface, they desorb due to a high desorption rate of $\mathrm{Ga}$ on $\mathrm{Si}$. This stability difference of Ga may lead to the isolated pillar-like growth. To investigate the influence of substrate surface on the columnar growth, GaN was grown on as-heated $\mathrm{Si}$ and sapphire under the same growth conditions as the pillar-like growth. On the as-heated $\mathrm{Si}$, no GaN growth is obtained. The $\mathrm{GaN}$ seeds, which are observed on the nitrided $\mathrm{Si}$, are not formed. On the other hand, $\mathrm{GaN}$ growth on sapphire results in a continuous film with a flat and mirror-like surface. These results indicate that stability of $\mathrm{Ga}$ on $\mathrm{Si}$ is improved by nitridation, and $\mathrm{Ga}$ adsorbs more stably on sapphire than nitrided $\mathrm{Si}$, leading to the growth of a single crystal film over the substrate. However, Yoshizawa et al. ${ }^{8}$ reported the columnar growth even on a sapphire substrate. This discrepancy probably arises from the difference of the growth conditions, such as the buffer layer formation, source materials, etc. Under our growth conditions, the hexagonal pillar-like growth is peculiar to the growth on a nitrided Si substrate. scale.
Downloaded 10 Jul 2008 to 133.70.80.50. Redistribution subject to AlP license or copyright; see http://apl.aip.org/apl/copyright.jsp

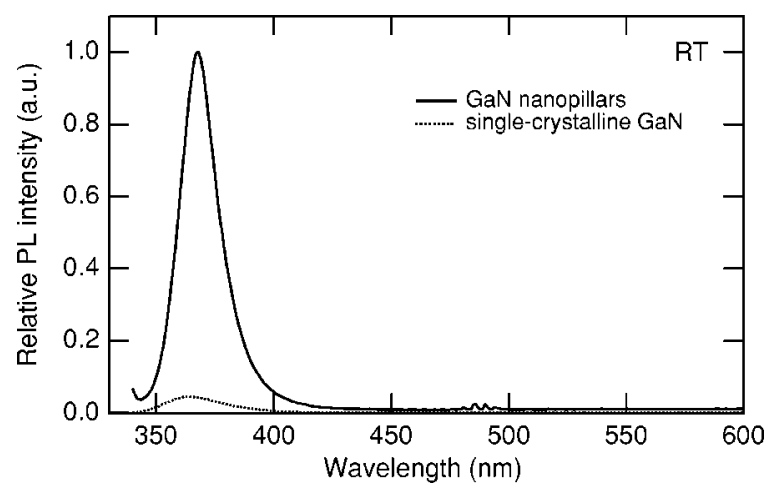

FIG. 2. PL spectra of the GaN nanopillars and the single-crystalline GaN at room temperature.

Figure 2 compares the room temperature PL spectra of the GaN nanopillars and the single-crystalline GaN grown on a sapphire substrate by HWE. For both samples, the nearband-edge emissions at $367 \mathrm{~nm}$ are observed, while the wellknown yellow band emissions are not observed. Clearly, the PL intensity of the GaN pillars is very strong. Considering the excitation volume contributing to luminescence, it is interesting to note that stronger emission is obtained from the sparsely grown GaN nanopillars than the continuous singlecrystalline $\mathrm{GaN}$ which luminesces comparably to a metalorganic chemical vapor deposition-grown GaN film. Such PL results would appreciably indicate the high crystal quality of GaN nanopillars.

The CL spectra of the GaN nanopillars and singlecrystalline $\mathrm{GaN}$ measured at room temperature are shown in Fig. 3. In the CL measurements, the GaN nanopillars again show the strong near-band-edge emission with a slight peak shift to $370 \mathrm{~nm}$. Comparing the relative intensities between $\mathrm{CL}$ and PL among the samples, the CL of the singlecrystalline GaN becomes weaker than the PL. The relative peak intensity ratio of the $\mathrm{GaN}$ nanopillars to the singlecrystalline $\mathrm{GaN}$ becomes 200 in CL, although the ratio in PL is 22 . This means that the emission excited by electron beam is more effectively obtained from the GaN nanopillars than the single-crystalline $\mathrm{GaN}$. These results suggest that $\mathrm{GaN}$ nanopillars have advantages for the application of electronimpact devices.

The crystal quality of nanopillars was investigated by cross-sectional TEM observation, shown in Fig. 4. The dark hexagon, viewed at the center, corresponds to the top or bottom view of an occasionally fallen pillar in the viewing di-

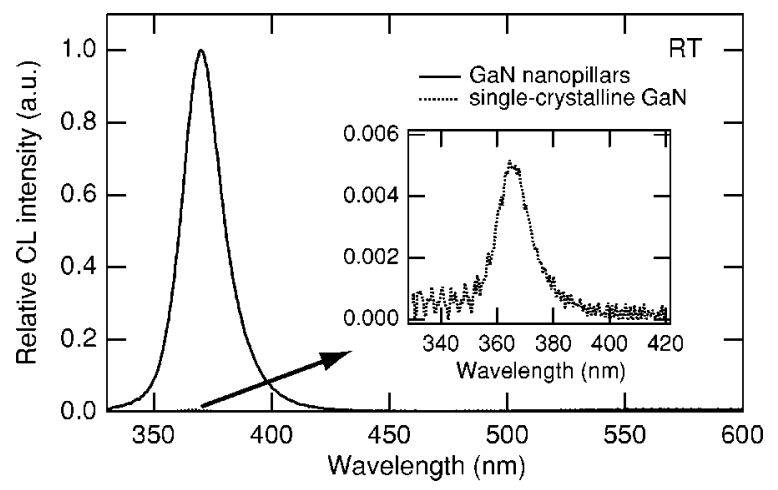

FIG. 3. CL spectra of the GaN nanopillars and the single-crystalline GaN. The inset shows the CL peak of the single-crystalline GaN on a magnified 


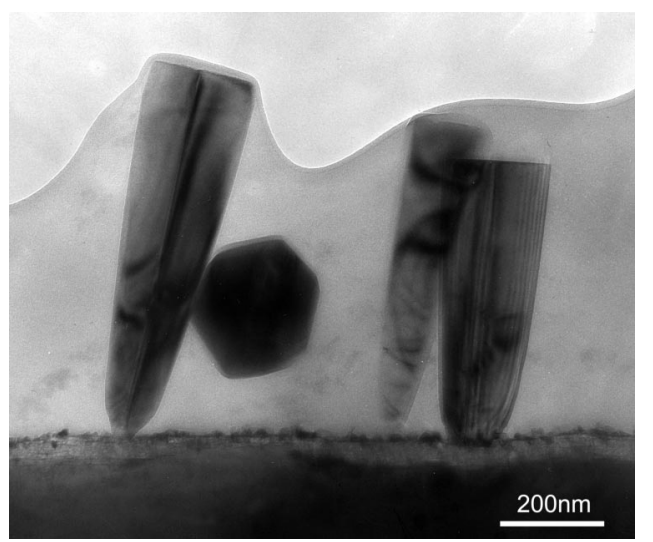

FIG. 4. Cross-sectional TEM image of the GaN nanopillars.

rection. Due to this occasion, we can clearly confirm the faceted hexagonal growth. The cross-sectional image, again, demonstrates that almost all the pillars grow with widening the diameter from $\sim 50$ to $\sim 200 \mathrm{~nm}$. We notice that the dark line at the center of the pillar corresponds to an edge of the hexagonal pillar, and fringes near the outer edge of pillars are due to interference of electron beam. Here, what is significant is that no dislocation could be observed for all of the pillars. This dislocation-free growth of $\mathrm{GaN}$ should directly result in the high luminescence. A possible cause for such dislocation-free growth is as follows. First, since the interface region between an initial $\mathrm{GaN}$ seed and $\mathrm{Si}$ is so small ( $\sim 50 \mathrm{~nm}$ in diameter), initial $\mathrm{GaN}$ seeds can grow without dislocations even with high strain. Then following the tiny $\mathrm{GaN}$ seeds, GaN pillars independently grow in the $c$-axis direction without coalescing with each other. Thereby, dislocations may not exist in nanopillars.

In addition, the structural effect might also play an important role on the strong light emissions from nanopillars. Since diameters of $\mathrm{GaN}$ nanopillars are as small as 200-300 nm, if the emitted light is reflected at the top sur- face of nanopillars, it can be extracted through side facets with some reflections. Therefore, this nanotexture effect of pillar-like structure will enhance the light extraction efficiency.

In this letter, we have demonstrated the growth of isolated dislocation-free $\mathrm{GaN}$ nanopillars on $\mathrm{Si}(111)$ substrates by a HWE technique. Both PL and CL for the GaN nanopillars show the strong near-band-edge emissions. The strong $\mathrm{CL}$ is observed from the $\mathrm{GaN}$ nanopillars rather than the single-crystalline $\mathrm{GaN}$, and is attributed to its high crystal quality and structural features. Such strong and effective CL emission suggests the possibility of fabrication of the electron-impact devices using III-nitrides.

This work was supported by a Grant-in-Aid of Japan Society for the Promotion of Science (Project No. 15560274), and Foundation for Promotion of Material Science and Technology of Japan.

${ }^{1}$ H. Kanie, T. Kawano, K. Sugimoto, and R. Kawai, Mater. Res. Soc. Symp. Proc. 621, Q5.11.1 (2000).

${ }^{2}$ K. Yamada, H. Asahi, H. Tampo, Y. Imanishi, K. Ohnishi, and K. Asami, Appl. Phys. Lett. 78, 2849 (2001).

${ }^{3}$ M. A. Sanchez-Garcia, E. Calleja, E. Monroy, F. J. Sanchez, F. Calle, E. Munoz, and R. Beresford, J. Cryst. Growth 183, 23 (1998).

${ }^{4}$ E. Calleja, M. A. Sanchez-Garcia, F. J. Sanchez, F. Calle, F. B. Naranjo, E. M. Munoz, U. Jahn, and K. Ploog, Phys. Rev. B 62, 16826 (2000).

${ }^{5}$ A. Ishida, T. Ose, H. Nagasawa, Y. Inoue, H. Fujiyasu, H. Makino, and T. Yao, Phys. Status Solidi C 0, 520 (2002).

${ }^{6}$ Y. Inoue, H. Nagasawa, N. Sone, K. Ishino, A. Ishida, H. Fujiyasu, J. J. Kim, H. Makino, T. Yao, S. Sakakibara, and M. Kuwabara, J. Cryst. Growth 265, 65 (2004).

${ }^{7}$ S. Sakakibara, F. Tanoue, M. Ohbora, M. Kaneko, T. Nakayama, K. Ishino, A. Ishida, and H. Fujiyasu, Appl. Surf. Sci. 142, 362 (1999).

${ }^{8}$ M. Yoshizawa, A. Kikuchi, M. Mori, N. Fujita, and K. Kishino, Jpn. J. Appl. Phys., Part 2 36, L459 (1997).

${ }^{9}$ S. Guha, N. A. Bojarczuk, M. A. L. Johonson, and J. F. Schetzina, Appl. Phys. Lett. 75, 463 (1999).

${ }^{10}$ H. M. Kim, D. S. Kim, D. Y. Kim, T. W. Kang, Y. H. Cho, and K. S. Chung, Appl. Phys. Lett. 81, 2193 (2002). 